\begin{tabular}{|c|l|}
\hline Title & The inverse scattering problem for Schrödinger and Klein-Gordon equations with a nonlocal nonlinearity \\
\hline Author(s) & Sasaki, Hironobu \\
\hline Citation & Hokkaido University Preprint Series in Mathematics, 772, 1-13 \\
\hline Issue Date & 2006 \\
\hline DOI & 10.14943/83922 \\
\hline Doc URL & http://hdl.handle.net/2115/69580 \\
\hline Type & bulletin (article) \\
\hline File Information & pre772.pdf \\
\hline
\end{tabular}

Instructions for use 


\title{
THE INVERSE SCATTERING PROBLEM FOR SCHRÖDINGER AND KLEIN-GORDON EQUATIONS WITH A NONLOCAL NONLINEARITY
}

\author{
HIRONOBU SASAKI* \\ DEPARTMENT OF MATHEMATICS, HOKKAIDO UNIVERSITY, \\ 060-0810, JAPAN.
}

\begin{abstract}
We study the inverse scattering problem for the nonlinear Schrödinger equation and for the nonlinear Klein-Gordon equation with the generalized Hartree type nonlinearity. We reconstruct the nonlinearity from knowledge of the scattering operator, which improves the known results.
\end{abstract}

\section{INTRODUCTION}

We consider the inverse scattering problem for the nonlinear Schrödinger equation

$$
i \partial_{t} u+\Delta u=f(u)
$$

and for the nonlinear Klein-Gordon equation

$$
\partial_{t}^{2} w-\Delta w+w=f(w)
$$

in space-time $\mathbb{R} \times \mathbb{R}^{n}$. Here $u$ is a complex-valued function of $(t, x) \in \mathbb{R} \times \mathbb{R}^{n}, w$ is either a complex-valued or real-valued function, $\partial_{t}=\partial / \partial t$ and $\Delta$ is the Laplacian in $\mathbb{R}^{n}$. The nonlocal nonlinear term $f(v)$ has the form

$$
f(v)=\int_{\mathbb{R}^{n}} \mu(x, y)|v(x-y)|^{2} v(x) d y .
$$

In order to state the condition of $\mu$, we define $A_{\sigma}^{l}$ for $\sigma \in(0, n)$ and $l=1,2, \cdots$ as the set of all functions $\nu:\left[\mathbb{R}^{n} \backslash\{0\}\right]^{2} \mapsto \mathbb{R}$ satisfying the following conditions:

(1) For $y \in \mathbb{R}^{n} \backslash\{0\}, \nu(\cdot, y) \in C^{l}\left(\mathbb{R}_{x}^{n} \backslash\{0\}\right)$ and for $x \in \mathbb{R}^{n} \backslash\{0\}, \nu(x, \cdot)$ is measurable on $\mathbb{R}_{y}^{n} \backslash\{0\}$.

(2) For $(x, y) \in\left[\mathbb{R}^{n} \backslash\{0\}\right]^{2},\left|\partial_{x}^{\alpha} \nu(x, y)\right| \leq C_{0}|y|^{-\sigma}$, where $C_{0}$ is independent of $x, y$ and $0 \leq|\alpha| \leq l$.

(3) There exists $\lambda_{0} \in C\left(\left[\mathbb{R}^{n} \backslash\{0\}\right]^{2}\right)$ such that

2000 Mathematics Subject Classification. 35R30, 35P25, 35Q40.

Key words and phrases. Inverse scattering, Scattering, Hartree type nonlinearity.

${ }^{*}$ Supported by Research Fellowships of the Japan Society for the Promotion of Science for Young Scientists. 
- $\lambda_{0}$ is bounded on $\left[\mathbb{R}^{n} \backslash\{0\}\right]^{2}$

- $\lambda_{0}$ is not 0 -function.

- $\lambda_{0}$ satisfies that either $\lambda_{0} \geq 0$ or $\lambda_{0} \leq 0$.

- $\lim _{\alpha \downarrow 0} \nu(\alpha x, \alpha y)|\alpha y|^{\sigma}=\lambda_{0}(x, y) \quad$ a.e.

Suppose that $\mu$ belongs to $A_{\sigma}^{1}$ with unknown $\sigma$.

The nonlinear term $f(v)$ is the generalization of the Hartree term

$$
G_{0}^{\sigma}(v)=\lambda\left(|\cdot|^{-\sigma} *|v|^{2}\right) v, \quad \lambda \in \mathbb{R} \backslash\{0\} .
$$

The term $G_{0}^{\sigma}$ is an approximative expression of the nonlocal interaction of specific elementary particles. The equations (NLS) and (NLKG) with $f=G_{0}^{\sigma}$ are initially studied by Chadam-Glassey [3] and Menzala-Strauss [8], respectively. There is a substantial literature on the scattering theory for Hartree equations (see for instance [11] and references therein).

The inverse scattering problem for the nonlinear equation is recovering the nonlinearity from the knowledge of the scattering operator. For the definition of the scattering operator for the nonlinear equation, see, e.g., Section 2 in [14]. As we will show later, under suitable conditions, the scattering operator is well-defined for (NLS) and for (NLKG).

The inverse scattering problem for the nonlinear Schrödinger equation with the Hartree term is initially studied by [17]. To introduce the other results, we define the following two terms:

$$
\begin{aligned}
& G_{1}(v)=\left(\lambda_{1}(\cdot)|\cdot|^{-\sigma} *|v|^{2}\right) v, \\
& G_{2}(v)=\lambda_{2}(x)\left(|\cdot|^{-\sigma} *|v|^{2}\right) v,
\end{aligned}
$$

where $\lambda_{j} \in C^{1}\left(\mathbb{R}^{n}\right) \cap W_{\infty}^{1}\left(\mathbb{R}^{n}\right), \lambda_{j}(0) \neq 0, j=1,2$. We remark that the term $G_{1}$ and $G_{2}$ satisfy (1.1) with $\mu=\lambda_{1}(y)|y|^{-\sigma} \in A_{\sigma}^{1}$ and $\mu=\lambda_{2}(x)|y|^{-\sigma} \in A_{\sigma}^{1}$, respectively. Watanabe [19] determined $\sigma$ of the term $G_{1}$ if $\lambda_{1}$ is a constant. However, the method of [19] is not applicable in the case where $\lambda_{1}$ is not constant. It was suggested in [19] that we can easily reconstruct the term $\lambda_{2}$ if $\sigma$ is a given number. Thus, if we can make a formula for determining $\sigma$ of $G_{2}$, then we can reconstruct $G_{2}$. However, the method to determine $\sigma$ of $G_{2}$ is not known in the case where $\lambda_{2}$ is not a constant.

The inverse scattering problem for the nonlinear Klein-Gordon equation with the Hartree term is initially studied by [14] which proved the uniqueness on identifying $\mu=\mu(y)$. We remark that for $\lambda>0$ with $\lambda \neq 1$, and for any nontrivial solution of a free Klein-Gordon equation $\phi(t, x)$, a rescaled function $\phi(\lambda t, \lambda x)$ does not solve the equation. As a result, the method to make the reconstruction formula for $G_{0}^{\sigma}$ in (NLS) is not applicable to the same term in (NLKG).

Our aim in this paper is to give an almost complete answer of the above problem which have not been shown. More precisely, for (NLS) and (NLKG), and for $j=1,2$, we shall determine $\sigma$ of $G_{j}$ even if $\lambda_{j}$ is not constant. Since $f$ with $\mu \in A_{\sigma}^{1}$ is a generalization of 
$G_{j}$, it is sufficient to make the formula for determining $\sigma$ of $\mu \in A_{\sigma}^{1}$.

To state our results, we give some notation. Let $\langle x\rangle=\left(1+|x|^{2}\right)^{1 / 2}$. For $s, k \in \mathbb{R}$, let $H_{p}^{s}$ and $H^{s, k}$ be the Sobolev space $(1-\Delta)^{-s / 2} L_{p}\left(\mathbb{R}^{n}\right)$ and the weighted Sobolev space $(1-\Delta)^{-s / 2}\langle x\rangle^{-k} L_{2}\left(\mathbb{R}^{n}\right)$, respectively. Especially, $H^{s}$ denotes $H_{2}^{s}$. For $1 \leq r \leq \infty$, let $\dot{r}$ be the Hölder conjugate of $r$. For $\alpha>0$ and $\phi: \mathbb{R}^{n} \rightarrow \mathbb{C}$, we denote $\phi\left(\alpha^{-1} x\right)$ by $\phi_{\alpha}(x)$. For (NLS), we set

$$
T[\phi]=\lim _{\varepsilon \downarrow 0} \frac{i}{\varepsilon^{3}}\langle(S-I)(\varepsilon \phi), \phi\rangle_{L^{2}\left(\mathbb{R}^{n}\right)},
$$

where $S$ is the scattering operator for (NLS). For (NLKG), we put

$$
K[\phi]=\lim _{\varepsilon \downarrow 0} \frac{i}{\varepsilon^{3}}\left\langle(S-I)\left(\varepsilon^{t}(\phi, 0)\right),{ }^{t}(0, \phi)\right\rangle_{H^{1}\left(\mathbb{R}^{n}\right) \oplus L_{2}\left(\mathbb{R}^{n}\right)},
$$

where $S$ is the scattering operator for (NLKG).

Again we assume that the nonlinearity $f$ has the form (1.1), and $\mu$ belongs to $A_{\sigma}^{1}$ with unknown $\sigma$. We are in a position to state our main Theorems.

Theorem 1.1. Let $n \geq 2,1<\sigma \leq 4, \sigma<n$. Assume that $\phi \in H^{1} \cap H^{0,1}$ and $\phi \neq 0$. For $(N L S)$, we have the formula for determining $\sigma$

$$
\sigma=2 n+2-\lim _{\alpha \downarrow 0} \ln \frac{\left|T\left[\phi_{e \alpha}\right]\right|}{\left|T\left[\phi_{\alpha}\right]\right|+\alpha^{2 n+2}} .
$$

Theorem 1.2. Let $I=(6(n-1) /(3 n-5), 2 n /(n-2)]$. Suppose that $n \geq 3, \max \{n /(n-$ $1), 4 / 3\}<\sigma \leq 4, \sigma<n$,

$$
\phi \in H^{1,1 / 3} \cap \bigcap_{r \in I} H_{\dot{r}}^{(n+1)(1 / 2-1 / r)}
$$

and $\phi \neq 0$. For $(N L K G)$, we have the formula for determining $\sigma$

$$
\sigma=2 n+1-\lim _{\alpha \downarrow 0} \ln \frac{\left|K\left[\phi_{e \alpha}\right]\right|}{\left|K\left[\phi_{\alpha}\right]\right|+\alpha^{2 n+1}} .
$$

Remark 1. From Theorem 1.1 or 1.2 , we can determine $\sigma$ of $G_{1}$ and of $G_{2}$. Moreover, by using the determined $\sigma$ and the method of [15] (see also (1.6) in [19]), we have the reconstruction formula for $\lambda_{1}$ of (NLS)

$$
\lambda_{1}\left(x_{0}\right)=\frac{\lim _{\alpha \rightarrow 0} \alpha^{-(2 n+2-\sigma)} T\left[\phi_{\alpha, x_{0}}\right]}{\int|y|^{-\sigma}\left|u_{0}(t, x-y)\right|^{2}\left|u_{0}(t, x)\right|^{2} d(t, x, y)} .
$$

Here, $\phi_{\alpha, x_{0}}(x)$ denotes $\phi\left(\alpha^{-1}\left(x-x_{0}\right)\right)$, and $u_{0}=e^{i t \Delta} \phi$. For (NLKG), the reconstruction formula for $\lambda_{1}$ can be also given by

$$
\lambda_{1}\left(x_{0}\right)=\frac{\lim _{\alpha \rightarrow 0} \alpha^{-(2 n+1-\sigma)} K\left[\phi_{\alpha, x_{0}}\right]}{\int|y|^{-\sigma}\left|w_{0}(t, x-y)\right|^{2}\left|w_{0}(t, x)\right|^{2} d(t, x, y)},
$$


where $w_{0}=\cos (t \sqrt{-\Delta}) \phi$. For the proof of (1.5), see Section 3.2.

Remark 2. In [19], The condition for $\sigma$ of the equation (NLS) is $2 \leq \sigma \leq 4$ and $\sigma<n$. We extend this condition to $1<\sigma \leq 4$ and $\sigma<n$.

The contents of this paper are as follows. In Section 2, we prove that we can treat $\mu \in$ $A_{\sigma}^{1}$ as $|y|^{-\sigma}$ whenever we consider only the scattering problems for (NLS) and for (NLKG). For this purpose, we first give an estimate for the nonlinearity by using the HardyLittlewood-Sobolev inequality and the complex interpolation method for the Sobolev spaces. From the estimate, we see the existence of the scattering operators for (NLS) and for (NLKG) under suitable condition of $\sigma$.

In Section 3, we first determine $\sigma$ of (NLS). As the first step, by using the method of [15] and the proposition in Section 2, we show that $\left|T\left[\phi_{e \alpha}\right]\right| /\left(\left|T\left[\phi_{\alpha}\right]\right|+\alpha^{2 n+2}\right)$ is nearly equal to

$$
e^{2 n+2-\sigma} \frac{\int \mu(e \alpha x, e \alpha y)|e \alpha y|^{\sigma} R(\phi)(t, x, y) d(t, x, y)}{\int \mu(\alpha x, \alpha y)|\alpha y|^{\sigma} R(\phi)(t, x, y) d(t, x, y)},
$$

where a function $R(\phi)(t, x, y)$ is integrable on $\mathbb{R}_{(t, x, y)}^{1+n+n}$. The condition for $\mu$ and the Lebesgue dominate theorem enable us to show (1.2). We next determine $\sigma$ of (NLKG). As in the (NLS) case, we first prove that $\left|K\left[\phi_{e \alpha}\right]\right| /\left(\left|K\left[\phi_{\alpha}\right]\right|+\alpha^{2 n+1}\right)$ is nearly equal to

$$
e^{2 n+1-\sigma} \frac{\int \mu(e \alpha x, e \alpha y)|e \alpha y|^{\sigma} Q^{\alpha}(\phi)(t, x, y) d(t, x, y)}{\int \mu(\alpha x, \alpha y)|\alpha y|^{\sigma} Q^{\alpha}(\phi)(t, x, y) d(t, x, y)},
$$

where

$$
Q^{m}(t, x, y)=|y|^{\sigma}\left|\Psi^{m}(t, x-y)\right|^{2}\left|\Psi^{m}(t, x)\right|^{2},
$$

and

$$
\Psi^{m}(t)=\cos \left(t \sqrt{m^{2}-\Delta}\right) \phi .
$$

In order to complete the theorem, we have only to prove that $\int Q^{\alpha} \rightarrow \int Q^{0}$ as $\alpha \rightarrow 0$. To show the convergence, we use the $L^{p}-L^{q}$ estimates for the linear Klein-Gordon and for the linear wave equation. Finally, we show the reconstruction formula (1.5).

\section{SCATTERING}

Before considering the inverse scattering problem, we have to solve the direct problem. It has been proved that there exist the scattering operators for (NLS) and for (NLKG) with $f=G_{0}^{\sigma}$ (see, e.g., $[5,6,7,13,16]$ ).

The following proposition is helpful to consider the direct problem:

Proposition 2.1. Let $l=1,2, \cdots, 0 \leq s \leq l, 1<r_{1}<r<\infty, 0<\sigma<n$ and $1+1 / r=\sigma / n+1 / r_{1}$. If $\nu$ satisfies the conditions (1) and (2) in the definition of $A_{\sigma}^{l}$, 
then we have for any $g \in H_{r_{1}}^{s}$

$$
\left\|\int_{\mathbb{R}^{n}} \nu(\cdot, y) g(\cdot-y) d y\right\|_{H_{r}^{s}} \leq C\|g\|_{H_{r_{1}}^{s}} .
$$

Proof. Let $0 \leq|\alpha| \leq l$. Since $\left|\partial_{x}^{\alpha} \nu(x, y)\right| \leq C_{0}|y|^{-\sigma}$, it follows from the Hardy-LittlewoodSobolev inequality and the Hölder inequality that

$$
\begin{aligned}
& \left\|\partial_{x}^{\alpha} \int_{\mathbb{R}^{n}} \nu(\cdot, y) g(\cdot-y) d y\right\|_{L_{r}} \\
& \quad \leq C \sum_{\alpha=\beta+\gamma}\left\|\int_{\mathbb{R}^{n}}\left(\partial_{x}^{\beta} \nu(\cdot, y)|y|^{\sigma}\right)|y|^{-\sigma} \partial_{x}^{\gamma} g(\cdot-y) d y\right\|_{L_{r}} \\
& \quad \leq C \sum_{\alpha=\beta+\gamma}\left\|\int_{\mathbb{R}^{n}}|y|^{-\sigma} \partial_{x}^{\gamma} g(\cdot-y) d y\right\|_{L_{r}} \\
& \quad \leq C \sum_{\gamma \leq \alpha}\left\|\partial_{x}^{\gamma} g\right\|_{L_{r_{1}}} .
\end{aligned}
$$

In particular, we have

$$
\begin{gathered}
\left\|\int_{\mathbb{R}^{n}} \nu(\cdot, y) g(\cdot-y) d y\right\|_{L_{r}} \leq C\|g\|_{L_{r_{1}}}, \\
\left\|\int_{\mathbb{R}^{n}} \nu(\cdot, y) g(\cdot-y) d y\right\|_{H_{r}^{l}} \leq C\|g\|_{H_{r_{1}}^{l}} .
\end{gathered}
$$

Accordingly, by using the complex interpolation method (see, e.g., [2]) for the linear operator $L_{r_{1}} \ni g \mapsto \int \nu(\cdot, y) g(\cdot-y) d y \in L_{r}$, we obtain $(2.1)$.

From (2.1), and the methods of $[5,6,13]$, we can see the existence of the scattering operator for some $\sigma$. In order to mention the scattering states in detail, we list some notation. For a Banach space $A$, and for $\delta>0$, let $B(\delta ; A)$ be the set $\{a \in A ;\|a \mid A\| \leq \delta\}$. Put $\omega=\sqrt{1-\Delta}$

$$
U(t)=\left(\begin{array}{cc}
\cos (t \omega) & \omega^{-1} \sin (t \omega) \\
-\omega \sin (t \omega) & \cos (t \omega)
\end{array}\right)
$$

and

$$
f\left(v_{1}, v_{2}, v_{3}\right)=\int_{\mathbb{R}^{n}} \mu(x, y) v_{1}(x-y) \overline{v_{2}(x-y)} v_{3}(x) d y
$$

We set

$$
Y_{\sigma}^{1}=\left\{\begin{array}{cc}
H^{1} \cap H^{0,1} & \text { for } 1<\sigma<2 \\
H^{1} & \text { for } 2 \leq \sigma \leq 4
\end{array}\right.
$$


and

$$
Y_{\sigma}^{2}=\left\{\begin{array}{cc}
H^{1, \frac{1}{3}} \oplus H^{0, \frac{1}{3}} & \text { for } 4 / 3<\sigma<2 \\
H^{1} \oplus L_{2} & \text { for } 2 \leq \sigma \leq 4
\end{array}\right.
$$

For a Banach space $A$, we denote $L^{p}(\mathbb{R} ; A)$ by $L^{p} A$. Let

$$
Z_{\sigma}^{1}=\left\{\begin{array}{cc}
B_{1} & \text { for } 1<\sigma \leq 4 / 3 \\
B_{2} & \text { for } 4 / 3<\sigma<2 \\
L^{3} H_{q_{1}}^{1} & \text { for } 2 \leq \sigma \leq 4
\end{array}\right.
$$

where

$$
\begin{aligned}
& B_{1}=\left\{e^{-i t \Delta} u(t) \in C\left(\mathbb{R} ; Y_{\sigma}^{1}\right) ; \sup _{t \in \mathbb{R}}\left\|e^{-i t \Delta} u(t)\right\|_{Y_{\sigma}^{1}}<\infty\right\}, \\
& B_{2}=\left\{e^{-i t \Delta} u(t) \in C\left(\mathbb{R} ; Y_{\sigma}^{1}\right), \omega u \in L^{8 / \sigma} L^{\tilde{q}}, e^{i t \Delta} x^{\beta} e^{-i t \Delta} u \in L^{8 / \sigma} L^{\tilde{q}} ;|\beta| \leq 1,\right. \\
& \left.\sup _{t \in \mathbb{R}}\left\|e^{-i t \Delta} u(t)\right\|_{Y_{\sigma}^{1}}+\|\omega u\|_{L^{8 / \sigma} L^{\tilde{q}}}+\sum_{|\beta| \leq 1}\left\|e^{i t \Delta} x^{\beta} e^{-i t \Delta} u\right\|_{L^{8 / \sigma} L^{\tilde{q}}}<\infty\right\},
\end{aligned}
$$

$1 / q_{1}=1 / 2-2 / 3 n, 1 / \tilde{q}=1 / 2-\sigma / 8$. Further, we define

$$
Z_{\sigma}^{2}=\left\{\begin{array}{cc}
L^{p_{2}} H_{q_{2}}^{1-\rho_{2}} & \text { for } 4 / 3<\sigma<2 \\
L^{3} H_{q_{3}}^{1-\rho_{3}} & \text { for } 2 \leq \sigma \leq 4
\end{array}\right.
$$

where

$$
\rho_{2}=\max \left\{\frac{n+2}{n}\left(1-\frac{1}{p_{2}}\right), \frac{2-\sigma}{4}\right\}, \quad \rho_{3}=\frac{n+1+\theta}{3(n-1+\theta)},
$$

$1 / q_{3}=1 / 2-2 / 3(n-1+\theta), \theta=2-\sigma / 2$. Here, $p_{2}$ and $q_{2}$ satisfy that $3<p_{2}<\infty$, $2<q_{2}<\infty$,

$$
\frac{n}{2}\left(\frac{1}{2}-\frac{1}{q_{2}}\right)<\frac{1}{p_{2}}<n\left(\frac{1}{2}-\frac{1}{q_{2}}\right), \quad \sigma=2-2\left\{\frac{2}{p_{2}}-n\left(\frac{1}{2}-\frac{1}{q_{2}}\right)\right\} .
$$

The scattering states are as follows:

Theorem 2.2. Assume that $\mu$ belongs to $A_{\sigma}^{1}$ with $0<\sigma<n$. Then we have the following properties: 
(1) Let $n \geq 2,1<\sigma \leq 4$ and $\sigma<n$. There exists some $\rho_{1}>0$ such that for any $\phi_{-} \in B_{\rho_{1}}\left(Y_{\sigma}^{1}\right)$, we uniquely have $u \in Z_{\sigma}^{1}$ and $\phi_{+} \in H^{1}$ such that

$$
\begin{aligned}
& u \in C\left(\mathbb{R} ; H^{1}\right), \\
& u(t)=e^{i t \Delta} \phi_{-}+\frac{1}{i} \int_{-\infty}^{t} e^{i(t-\tau) \Delta} f(u(\tau)) d \tau, \\
& \left\|u(t)-e^{i t \Delta} \phi_{ \pm}\right\|_{H^{1}} \rightarrow 0 \quad \text { as } t \rightarrow \pm \infty \\
& \|u\|_{Z_{\sigma}^{1}} \leq C\left\|\phi_{-}\right\|_{Y_{\sigma}^{1}}, \\
& \left\|u-e^{i t \Delta} \phi_{-}\right\|_{Z_{\sigma}^{1}} \leq C\left\|\phi_{-}\right\|_{Y_{\sigma}^{1}}^{3}, \\
& \left\|\int_{\mathbb{R}} e^{-i t \Delta} f\left(u_{1}, u_{2}, u_{3}\right) d t\right\|_{L_{2}} \leq C \Pi_{j=1}^{3}\left\|u_{j}\right\|_{Z_{\sigma}^{1}} \\
& \quad \text { for } u_{j} \in Z_{\sigma}^{1}, j=1,2,3 .
\end{aligned}
$$

Moreover, we can define the scattering operator for (NLS)

$$
S: B_{\rho_{1}}\left(Y_{\sigma}^{1}\right) \ni \phi_{-} \mapsto \phi_{+}=\phi_{-}+\frac{1}{i} \int_{\mathbb{R}} e^{-i t \Delta} f(u(t)) d t \in H^{1} .
$$

(2) Let $n \geq 2,4 / 3<\sigma \leq 4$ and $\sigma<n$. There exists some $\rho_{2}>0$ such that for any $\psi_{-}={ }^{t}\left(\psi_{-}^{1}, \psi_{-}^{2}\right) \in B_{\rho_{2}}\left(Y_{\sigma}^{2}\right)$, we uniquely have $w \in Z_{\sigma}^{2}$ and $\psi_{+} \in H^{1} \oplus L_{2}$ such that

$$
\begin{aligned}
& W={ }^{t}\left(w, \partial_{t} w\right) \in C^{1}\left(\mathbb{R} ; H^{1}\right) \oplus C\left(\mathbb{R} ; L_{2}\right) \\
& W(t)=U(t) \phi_{-}+\frac{1}{i} \int_{-\infty}^{t} U(t-\tau)\left(\begin{array}{c}
0 \\
i f(w(\tau))
\end{array}\right) d \tau \\
& \left\|W(t)-U(t) \psi_{ \pm}\right\|_{H^{1} \oplus L_{2}} \rightarrow 0 \quad \text { as } t \rightarrow \pm \infty \\
& \|w\|_{Z_{\sigma}^{2}} \leq C\left\|\psi_{-}\right\|_{Y_{\sigma}^{2}}, \\
& \left\|w-\left(\cos (t \omega) \psi_{-}^{1}+\omega^{-1} \sin (t \omega) \psi_{-}^{2}\right)\right\|_{Z_{\sigma}^{2}} \leq C\left\|\psi_{-}\right\|_{Y_{\sigma}^{2}}^{3}, \\
& \left\|\int_{\mathbb{R}} e^{i t \omega} f\left(w_{1}, w_{2}, w_{3}\right) d t\right\|_{L_{2}} \leq C \Pi_{j=1}^{3}\left\|w_{j}\right\|_{Z_{\sigma}^{2}} \\
& \quad \text { for } w_{j} \in Z_{\sigma}^{1}, j=1,2,3 .
\end{aligned}
$$

Moreover, we can define the scattering operator for (NLKG)

$$
S: B_{\rho_{2}}\left(Y_{\sigma}^{2}\right) \ni \psi_{-} \mapsto \psi_{+}=\psi_{-}+\frac{1}{i} \int_{\mathbb{R}} U(-t)\left(\begin{array}{c}
0 \\
i f(w(t))
\end{array}\right) d t \in H^{1} \oplus L_{2} .
$$

Proof. In the case of both (NLS) and (NLKG) with $2 \leq \sigma \leq 4$, the estimate (2.1) enables us to see that (3.6) and (3.7) in [6] hold.

In the case of (NLS) with $1<\sigma<2$, the estimates $(2,5)-(2,8)$ in [5] are immediately shown by $(2.1)$. 
In the case of (NLKG) with $4 / 3<\sigma<2$, Lemma 2.3 in [13] holds from (2.1).

Accordingly, we can treat the estimates for $f$ as the estimates for the Hartree type $G_{0}^{\sigma}$. For the rest of the proof, we have only to apply to methods of $[5,6,13]$. This completes the proof.

\section{INVERSE SCATTERING}

In this section, we consider the inverse scattering problem for (NLS) and for (NLKG).

3.1. Nonlinear Schrödinger equation. Inverse scattering problem for the nonlinear Schrödinger equation was initially studied by [15]. Weder [21, 22, 24] considered the nonlinear Schrödinger equation with a power type nonlinearity.

It was mentioned by [14] that the method for the power type nonlinearity is not applicable to our problem of determing $\mu(x, y)$ in (NLS).

Now we prove Theorem 1.1. Let $1<\sigma \leq 4$ and $\sigma<n$. We here assume $\mu \in A_{\sigma}^{1}$. From Theorem 2.2,(1), if $\phi \in H^{1} \cap H^{0,1}$, then $T[\phi]$ is well-defined.

By [15] and [18], it follows from (2.5)-(2.7) that

$$
T[\phi]=\int_{\mathbb{R}_{(t, x, y)}^{1+n+n}} \mu(x, y)\left|e^{i t \Delta} \phi(x-y)\right|^{2}\left|e^{i t \Delta} \phi(x)\right|^{2} d(t, x, y) .
$$

Having in mind that

$$
e^{i t \Delta} \phi_{\alpha}=\left(e^{i t \alpha^{-2} \Delta} \phi\right)_{\alpha}
$$

we see that

$$
T\left[\phi_{\alpha}\right]=\alpha^{2 n+2-\sigma} \int_{\mathbb{R}_{(t, x, y)}^{1+n+n}} \mu(\alpha x, \alpha y)|\alpha y|^{\sigma} R(\phi)(t, x, y) d(t, x, y),
$$

where

$$
R(\phi)(t, x, y)=|y|^{-\sigma}\left|e^{i t \Delta} \phi(x-y)\right|^{2}\left|e^{i t \Delta} \phi(x)\right|^{2} .
$$

Substituting $\mu(x, y)=|y|^{-\sigma}$ into (3.1), we see that $R(\phi)$ is integrable. Hence it follows from the assumption $\mu \in A_{\sigma}^{1}$ that

$$
\begin{aligned}
\int_{\mathbb{R}_{(t, x, y)}^{1+n+n}} \mu(\beta x, \beta y)|\beta y|^{\sigma} R(\phi)(t, x, y) d(t, x, y) . \\
\rightarrow \int_{\mathbb{R}_{(t, x, y)}^{1+n+n}} \lambda_{0}(x, y) R(\phi)(t, x, y) d(t, x, y) \neq 0 \quad \text { as } \beta \rightarrow 0
\end{aligned}
$$


if $\phi \neq 0$. Hence we obtain

$$
\begin{aligned}
\frac{\left|T\left[\phi_{e \alpha}\right]\right|}{\left|T\left[\phi_{\alpha}\right]\right|+\alpha^{2 n+2}}=\frac{\left.e^{2 n+2-\sigma}\left|\int \mu(e \alpha x, e \alpha y)\right| e \alpha y\right|^{\sigma} R(\phi)(t, x, y) \mid}{\left.\left|\int \mu(\alpha x, \alpha y)\right| \alpha y\right|^{\sigma} R(\phi)(t, x, y) \mid+\alpha^{\sigma}} & \\
\rightarrow e^{2 n+2-\sigma} \quad \text { as } \alpha & \rightarrow 0
\end{aligned}
$$

Thus, we have (1.2). This completes the proof.

3.2. Nonlinear Klein-Gordon equation. Inverse scattering problem for the nonlinear Klein-Gordon equation was initially studied by [9]. Weder [20, 23] (see also [1]) considered the nonlinear Klein-Gordon equation with a power type nonlinearity

$$
\frac{\partial^{2} w}{\partial t^{2}}(x, t)-\Delta w(x, t)+w(x, t)=V_{0}(x) w(x, t)+\sum_{j=1}^{\infty} V_{j}(x)|w|^{2\left(j_{0}+j\right)} w(x, t) .
$$

It was proved that the small amplitude limit of the scattering operator determines uniquely all the $V_{j}(x), j=0,1, \cdots$.

It was mentioned by [14] that the method for the power type nonlinearity is not applicable to our problem of determing $\mu(x, y)$ in (NLKG).

Now we prove Theorem 1.2, and the reconstruction formula (1.5). To derive (1.3), we follow the line of the proof of Theorem 1.1. Let $4 / 3<\sigma \leq 4, n /(n-1)<\sigma<n$ and let $S$ be the scattering operator for (NLKG).

We here assume that $\mu \in A_{\sigma}^{1}$,

$$
\phi \in \Lambda=H^{1,1 / 3} \cap \bigcap_{r \in I} H_{\dot{r}}^{(n+1)(1 / 2-1 / r)}
$$

and $\phi \neq 0$, where $I=(6(n-1) /(3 n-5), 2 n /(n-2)]$. By Theorem 2.2,(2), and $\Lambda \subset H^{1,1 / 3}$, $K[\phi]$ is well-defined. By (2.11)-(2.13), it follows from the method of [15] and [18] that

$$
K[\phi]=\int_{\mathbb{R}_{(t, x, y)}^{1+n+n}} \mu(x, y)\left|\Psi^{1}(t, x-y)\right|^{2}\left|\Psi^{1}(t, x)\right|^{2} d(t, x, y),
$$

where $\Psi^{m}(t)=\cos \left(t \sqrt{m^{2}-\Delta}\right) \phi$.

Having in mind that

$$
e^{i t \sqrt{1-\Delta}} \phi_{\alpha}=\left(e^{i t \alpha^{-1} \sqrt{\alpha^{2}-\Delta}} \phi\right)_{\alpha}
$$

we also have

$$
K\left[\phi_{\alpha}\right]=\alpha^{2 n+1-\sigma} \int_{\mathbb{R}_{(t, x, y)}^{1+n+n}} \mu(\alpha x, \alpha y)|\alpha y|^{\sigma} Q^{\alpha}(\phi)(t, x, y) d(t, x, y),
$$

where

$$
Q^{m}(\phi)(t, x, y)=|y|^{-\sigma}\left|\Psi^{m}(t, x-y)\right|^{2}\left|\Psi^{m}(t, x)\right|^{2} .
$$


In order to see the convergence of $\left|K\left[\phi_{e \alpha}\right]\right| /\left(\left|K\left[\phi_{\alpha}\right]\right|+\alpha^{2 n+1}\right)$, we give the following lemma:

Lemma 3.1. Let $I=(6(n-1) /(3 n-5), 2 n /(n-2)]$ and $0 \leq \beta \leq 1$. For $n /(n-1)<$ $\sigma \leq 4, \sigma<n$, and

$$
\phi \in H^{1} \cap \bigcap_{r \in I} H_{\dot{r}}^{(n+1)(1 / 2-1 / r)},
$$

then $Q^{\beta}(\phi)(t, x, y)$ is integrable on $\mathbb{R}_{(t, x, y)}^{1+n+n}$. Moreover, we have

$$
\int Q^{\beta}(\phi) d(t, x, y) \rightarrow \int Q^{0}(\phi) d(t, x, y) \quad \text { as } \beta \rightarrow 0 .
$$

Proof. We first state $L^{p}-L^{p}$ estimates (see, e.g., $[10,12]$ )

$$
\left\|e^{i t \sqrt{-\Delta}} \phi\right\|_{L^{r}} \leq C|t|^{-(n-1)(1 / 2-1 / r)}\|\phi\|_{\dot{H}_{\dot{r}}^{(n+1)(1 / 2-1 / r)}}
$$

and

$$
\left\|e^{i t \sqrt{1-\Delta}} \phi\right\|_{L^{r}} \leq C|t|^{-(n-1)(1 / 2-1 / r)}\|\phi\|_{H_{\dot{r}}^{(n+1)(1 / 2-1 / r)}},
$$

where $2<r<\infty$ and $\dot{H}_{p}^{s}$ is the homogeneous Sobolev space (for the definition, see, e.g., $[2,4])$.

By the embedding $H_{\dot{r}}^{s} \hookrightarrow \dot{H}_{\dot{r}}^{s}$ and $H^{n(1 / 2-1 / r)} \hookrightarrow L^{r}$, it follows from (3.3) that

$$
\left\|e^{i t \sqrt{-\Delta}} \phi\right\|_{L^{r}} \leq C\langle t\rangle^{-(n-1)(1 / 2-1 / r)} N_{r}(\phi),
$$

where

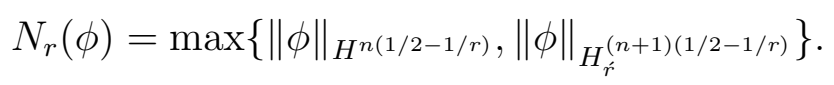

On the other hand, Using the identity

$$
e^{i t \sqrt{m^{2}-\Delta}} \phi=\left(e^{i t m \sqrt{1-\Delta}} \phi_{m}\right)_{m^{-1}},
$$

and (3.4), we obtain

$$
\begin{aligned}
&\left\|e^{i t \sqrt{m^{2}-\Delta}} \phi\right\|_{L_{r}}=m^{-n / r}\left\|e^{i t m \sqrt{1-\Delta}} \phi_{m}\right\|_{L_{r}} \\
& \leq C m^{-n / r}|t m|^{-(n-1)(1 / 2-1 / r)}\left\|\phi_{m}\right\|_{H_{\dot{r}}^{(n+1)(1 / 2-1 / r)}} \\
& \leq \quad C m^{(n+1)(1 / 2-1 / r)}\left(1+m^{-(n+1)(1 / 2-1 / r)}\right) \\
& \times|t|^{-(n-1)(1 / 2-1 / r)}\|\phi\|_{H_{\dot{r}}^{(n+1)(1 / 2-1 / r)}} \\
& \leq C|t|^{-(n-1)(1 / 2-1 / r)}\|\phi\|_{H_{\dot{r}}^{(n+1)(1 / 2-1 / r)}}
\end{aligned}
$$

if $0<m \leq 1$. By the embedding $H^{n(1 / 2-1 / r)} \hookrightarrow L^{r}$, it follows from (3.5) that

$$
\left\|e^{i t \sqrt{\beta^{2}-\Delta}} \phi\right\|_{L^{r}} \leq C\langle t\rangle^{-(n-1)(1 / 2-1 / r)} N_{r}(\phi) .
$$


Let $s \in[0,1]$. By the Hardy-Littlewood-Sobolev inequality, the Hölder inequality and the embedding $L^{(1 / 2+s / n)^{-1}} \hookrightarrow H^{-s}$, we have

$$
\begin{aligned}
\| Q^{m}(\phi)- & Q^{0}(\phi) \|_{L^{1}\left(\mathbb{R}_{(x, y)}^{2 n}\right)} \\
\leq & \left\|\left(|\cdot|^{-\sigma} *\left(\Psi^{m}-\Psi^{0}\right) \overline{\Psi^{m}}\right) \Psi^{m} \overline{\Psi^{m}}\right\|_{L^{1}\left(\mathbb{R}^{n}\right)} \\
& +\left\|\left(|\cdot|^{-\sigma} * \Psi^{0}\left(\overline{\Psi^{m}-\Psi^{0}}\right)\right) \Psi^{m} \overline{\Psi^{m}}\right\|_{L^{1}\left(\mathbb{R}^{n}\right)} \\
& +\left\|\left(|\cdot|^{-\sigma} * \Psi^{0} \overline{\Psi^{0}}\right)\left(\Psi^{m}-\Psi^{0}\right) \overline{\Psi^{m}}\right\|_{L^{1}\left(\mathbb{R}^{n}\right)} \\
& +\left\|\left(|\cdot|^{-\sigma} * \Psi^{0} \overline{\Psi^{0}}\right) \Psi^{0}\left(\overline{\Psi^{m}-\Psi^{0}}\right)\right\|_{L^{1}\left(\mathbb{R}^{n}\right)} \\
\leq & \sum_{\beta_{1}, \beta_{2} \in\{0,1\}}\left\|\left(|\cdot|^{-\sigma} *\left|\Psi^{\beta_{1}}\right|^{2}\right)\left|\Psi^{\beta_{2}}\right|\left|\Psi^{m}-\Psi^{0}\right|\right\|_{L^{1}\left(\mathbb{R}^{n}\right)} \\
\leq & C\left\|\Psi^{m}-\Psi^{0}\right\|_{H^{s}} \sum_{\beta_{1}, \beta_{2} \in\{0,1\}}\left\|\left(|\cdot|^{-\sigma} *\left|\Psi^{\beta_{1}}\right|^{2}\right)\left|\Psi^{\beta_{2}}\right|\right\|_{H^{-s}} \\
\leq & C\left\|\Psi^{m}-\Psi^{0}\right\|_{H^{1}} \sum_{\beta_{1}, \beta_{2} \in\{0,1\}}\left\|\left(|\cdot|^{-\sigma} *\left|\Psi^{\beta_{1}}\right|^{2}\right)\left|\Psi^{\beta_{2}}\right|\right\|_{L^{\left(\frac{1}{2}+\frac{s}{n}\right)-1}} \\
\leq & C\left\|\Psi^{m}-\Psi^{0}\right\|_{H^{1}}\left\{\left\|\Psi^{m}\right\|_{L^{r}}^{3}+\left\|\Psi^{0}\right\|_{L^{r}}^{3}\right\},
\end{aligned}
$$

where we have used the equality

$$
\int\left(|\cdot|^{-\sigma} * v_{1}\right) v_{2}=\int\left(|\cdot|^{-\sigma} * v_{2}\right) v_{1}
$$

for the second inequality, and $r$ satisfies

$$
\frac{3}{2}+\frac{s}{n}=\frac{\sigma}{n}+\frac{3}{r}, \quad 2<r<\infty .
$$

Using (3.6), we see that

$$
\left\|Q^{m}(\phi)-Q^{0}(\phi)\right\|_{L^{1}\left(\mathbb{R}_{(x, y)}^{2 n}\right)} \leq C\langle t\rangle^{-3(n-1)(1 / 2-1 / r)}\left(N_{r}(\phi)\right)^{3}\left\|\Psi^{m}-\Psi^{0}\right\|_{L^{2}} .
$$

Since $n /(n-1)<\sigma \leq 4$ and $\sigma<n$, we can put $r \in I$. Thus, we have $n(1 / 2-1 / r) \leq 1$ and $-3(n-1)(1 / 2-1 / r)<-1$. Hence, $Q^{\beta}(\phi)$ is integrable, and the right hand side of (3.7) is bounded by some integrable function which is independent of $m$. For all $t \in \mathbb{R}$, we can easily show that

$$
\left\|\Psi^{m}(t)-\Psi^{0}(t)\right\|_{H^{s}} \rightarrow 0 \quad \text { as } m \rightarrow 0
$$

Thus, applying the Lebesgue dominate theorem on $\mathbb{R}_{t}$, we have (3.2). This completes the proof. 
Let us go back to the proof of Theorem 1.2. We again assume $\phi \in \Lambda$. From Lemma 3.1 , we see that

$$
\begin{aligned}
\int_{\mathbb{R}_{(t, x, y)}^{1+n+n}} \mu(\alpha x, \alpha y)|\alpha y|^{\sigma} Q^{\alpha}(\phi)(t, x, y) d(t, x, y) . & \\
& \rightarrow \int_{\mathbb{R}_{(t, x, y)}^{1+n+n}} \lambda_{0}(x, y) Q^{0}(\phi)(t, x, y) d(t, x, y) \neq 0 \quad \text { as } \alpha \rightarrow 0
\end{aligned}
$$

So we obtain

$$
\begin{array}{r}
\frac{\left|K\left[\phi_{e \alpha}\right]\right|}{\left|K\left[\phi_{\alpha}\right]\right|+\alpha^{2 n+1}}=\frac{\left.e^{2 n+1-\sigma}\left|\int \mu(e \alpha x, e \alpha y)\right| e \alpha y\right|^{\sigma} Q^{0}(\phi)(t, x, y) \mid}{\left.\left|\int \mu(\alpha x, \alpha y)\right| \alpha y\right|^{\sigma} Q^{0}(\phi)(t, x, y) \mid+\alpha^{\sigma}} \\
\rightarrow e^{2 n+1-\sigma} \quad \text { as } \alpha \rightarrow 0 .
\end{array}
$$

Thus, we have (1.3). This completes the proof of Theorem 1.2.

It remains to show the reconstruction formula (1.5). We put $\phi \in \Lambda$ and $\phi_{\alpha, x_{0}}=$ $\phi\left(\alpha^{-1}\left(x-x_{0}\right)\right)$. Then we have

$$
K\left[\phi_{\alpha, x_{0}}\right]=\alpha^{2 n+1-\sigma} \int_{\mathbb{R}_{(t, x, y)}^{1+n+n}} \lambda_{1}\left(\alpha x+x_{0}\right) Q^{\alpha}(\phi)(t, x, y) d(t, x, y) .
$$

Here, $\sigma$ is a known number which is determined by Theorem 1.2. Since $\lambda_{1}$ is bounded and continuous, it follows from Lemma 3.1 that

$$
\lim _{\alpha \rightarrow 0} \alpha^{-(2 n+1-\sigma)} K\left[\phi_{\alpha, x_{0}}\right]=\int_{\mathbb{R}_{(t, x, y)}^{1+n+n}} \lambda_{1}\left(x_{0}\right) Q^{0}(\phi)(t, x, y) d(t, x, y) .
$$

Thus, we have (1.5).

Acknowledgement. The author would like to acknowledge the helpful guidance and encouragement of Dr. M. Watanabe.

\section{REFERENCES}

[1] A. Bachelot, Inverse scattering problem for the nonlinear Klein-Gordon equation, in: C. Bardos, A. Damlamian, J. I. Díaz and J. Hernández (Eds.), Contributions to nonlinear partial differential equations, Res. Notes in Math., 89, Pitman, Boston, MA, 1983, pp. 7-15.

[2] J. Bergh and J. Löfström, Interpolation spaces, Grundlehren der Mathematischen Wissenschaften, Springer, Berlin, 1976.

[3] J. Chadam and R. Glassey, Global existence of solutions to the Cauchy problem for time dependent Hartree equations, J. Math. Phys., t. 16 (1975) 1122-1130

[4] J. Ginibre and G. Velo, The global Cauchy problem for the non linear Klein-Gordon equation, Math. Z. 189 (1985) 487-505.

[5] N. Hayashi and Y. Tsutsumi, Scattering theory for Hartree type equations, Ann. Inst. Henri Poincaré, Physique Theorique 46 (1987) 187-213. 
[6] K. Mochizuki, On small data scattering with cubic convolution nonlinearity, J. Math. Soc. Japan 41 (1989) 143-160.

[7] K. Mochizuki and T. Motai, On small data scattering for some nonlinear wave equations, in: T. Nishida, M. Mimura and H. Fujii (Eds.), Patterns and Waves -qualitative analysis of nonlinear differential equations -, Stud. Math. Appl., 18, North-Holland, Amsterdam, 1986, pp. 543-560.

[8] G. Menzala and W. Strauss, On a wave equation with a cubic convolution, J. Differential Equations 43 (1982) 93-105.

[9] C. Morawetz and W. Strauss, On a nonlinear scattering operator, Comm. Pure Appl. Math. 26 (1973) 47-54.

[10] M. Nakamura and T. Ozawa, The Cauchy problem for nonlinear Klein-Gordon equations in the Sobolev spaces, Publ. Res. Inst. Math. Sci. 37 (2001) 255-293.

[11] K. Nakanishi, T. Ozawa, Scattering problem for nonlinear Schrödinger and Hartree equations, Tosio Kato's method and principle for evolution equations in mathematical physics, Sūrikaisekikenkyūsho Kōkyūroku 1234 (2001) 105-112.

[12] H. Pecher, Nonlinear small data scattering for the wave and Klein-Gordon equation, Math. Z. 185 (1984) 261-270.

[13] H. Sasaki, Small data scattering for the Klein-Gordon equation with cubic convolution nonlinearity, to appear in Discrete Contin. Dynam. Systems.

[14] H. Sasaki and M. Watanabe, Uniqueness on identification of cubic convolution nonlinearity, J. Math. Anal. Appl. 309 (2005) 294-306.

[15] W. Strauss, Non linear scattering theory, in: J. A. Lavita and J.-P. Marchand (Eds.), Scattering Theory in Mathematical Physics, D. Reidel, Dordrecht-Holland / Boston 1974, pp. 53-78.

[16] W. Strauss, Nonlinear scattering theory at low energy: sequel, J. Funct. Anal. 43 (1981) 281-293.

[17] M. Watanabe, Inverse scattering for the nonlinear Schrödinger equation with cubic convolution nonlinearity, Tokyo J. math. 24 (2001) 59-67.

[18] M. Watanabe, Uniqueness in the inverse scattering problem for the Hartree type equation, Proc. Japan Acad. Ser A. 77 (2001) 143-146.

[19] M. Watanabe, Reconstruction of the Hartree-type nonlinearity, Inverse Problems 18 (2002) 14771481.

[20] R. Weder, Inverse scattering on the line for the nonlinear Klein-Gordon equation with a potential, J. Math. Anal. Appl. 252 (2000) 102-123.

[21] R. Weder, Inverse scattering for the nonlinear Schrödinger equation. Reconstruction of the potential and the nonlinearity, Math. Meth. Appl. Sci. 24 (2001), 245-254.

[22] R. Weder, Inverse scattering for the nonlinear Schrödinger equation II. Reconstruction of the potential and the nonlinearity in the multidiemnsional case, Proc. Amer. Math. Soc. 129 (2001), 3637-3645.

[23] R. Weder, Multidimensional inverse scattering for the nonlinear Klein-Gordon equation with a potential, J. Differential Equations 184 (2002) 62-77.

[24] R. Weder, Scattering for the forced non-linear Schrödinger equation with a potential on the half-line, Math. Meth. Appl Sci. 28 (2005), 1219-1236.

E-mail address: hisasaki@math.sci.hokudai.ac.jp 